\title{
El atomismo de Leibniz en De summa rerum (1676): una interpretación a la luz de la cosmología cartesiana ${ }^{1}$
}

\section{Leibniz's atomism in De summa rerum (1676): an interpretation in the light of Cartesian cosmology}

\author{
Rodolfo Fazio*
}

\begin{abstract}
Resumen: En el presente trabajo estudiamos la teoría atomista que Leibniz propone en De summa rerum (1676). Luego de explicar las razones que esgrime con vistas a introducir átomos materiales en la naturaleza, defendemos una doble hipótesis. En primer lugar, mostramos que el atomismo leibniziano recibe inspiración de la cosmología cartesiana. En segundo lugar, proponemos que esta teoría ha de comprenderse en el marco de sus reflexiones acerca de la sustancia corpórea y que es concebida con vistas a superar las dificultades que el período parisino presenta a su metafísica juvenil.
\end{abstract}

Palabras clave: Leibniz, atomismo, cuerpo, mente, sustancia.

Abstract: In this paper we study Leibniz's atomist theory in De summa rerum (1676). After explaining why he introduces material atoms in nature, we defend a double hypothesis. First, we show that Leibnizian atomism receives his inspirations from Cartesian cosmology. Second, we hold that this theory should be understood in the context of his reflections on corporeal substances and, furthermore, that it is conceived in order to overcome the inconveniences that the Parisian period present to his metaphysics of youth.

Keywords: Leibniz, atomism, body, mind, substance.

\footnotetext{
${ }^{1}$ Agradezco a Beatriz von Bilderling y Federico Raffo Quintana por sus comentarios al presente trabajo.

* Doctor en Filosofía por la Universidad de Buenos Aires, Argentina, y ayudante de Filosofía Moderna en la misma institución. Su principal área de investigación es la filosofía del siglo XVII, en especial estudia temas de metafísica y filosofía natural en Leibniz. Ha sido becario de la Universidad de Buenos Aires, del CONICET y del Servicio Alemán de Intercambio Académico (DAAD) en el Leibniz-Forschungsstelle de la Universidad de Münster. Dirección electrónica: rodolfofazio@gmail.com
} 


\section{Introducción ${ }^{2}$}

Los años que Leibniz vive en Paris (1672-1676) constituyen uno de los mayores puntos de inflexión en el desarrollo de su metafísica. Si bien las razones de ello no son manifiestas, el resultado que las investigaciones parisinas tienen en su pensamiento es innegable: en ese período Leibniz abandona su solución juvenil al problema del continuo y sienta las bases para su respuesta definitiva, crítica su propia concepción juvenil del movimiento y presenta su primera defensa de la relatividad general del mismo y, por último, deja a un lado su metafísica juvenil de los cuerpos para ensayar diversas propuestas alternativas. ${ }^{3}$ Dejando de lado las posibles causas de estos cambios, en la presente investigación nos interesamos por evaluar la propuesta atomista que Leibniz desarrolla durante su último año en la capital francesa en los escritos reunidos bajo el título De summa rerum (1676). En esta serie de textos el filósofo alemán somete a revisión los conceptos básicos de su ontología: desde Dios, el cuerpo y la mente, hasta el universo, el vacío y los átomos. ${ }^{4}$ Muchos de estos

${ }^{2}$ Utilizamos las siguientes ediciones para los textos de Leibniz: Leibniz, G. W., Sämtliche Schriften und Briefe, Hrsg. von der Berlin-Brandenburgischen Akademie der Wissenschaften und der Wissenschaften in Göttingen, Darmstadt-Leipzig-Berlin, , Akademie Verlag, 1923-sig (se cita como A seguido por un número romano que indica la serie y los siguientes números arábigos refieren al tomo y las páginas); Leibniz, G. W., Die philosophischen Schriften, ed. C. I. Gerhardt, 7 vols., Berlin, Wiedeman Buchhandlung, 1875-1890 (se cita como GP seguido por el tomo en números romanos y las páginas en números arábigos).

3 Sobre los dos primeros cambios y la implicancia que tiene para la metafísica leibniziana, véase Fazio, R., "La crítica de Leibniz a los números infinitos y su repercusión en la metafísica de los cuerpos", en Theoria: An International Journal for Theory, History and Foundations of Science, 31/2, 2016, pp. 159-175; Fazio, R., "Movimiento, cuerpo y sustancia corpórea en Leibniz: la defensa de la relatividad del movimiento y su impacto en el desarrollo de la metafísica de los cuerpos", Eidos, 26, 2017, pp. 238-267.

${ }^{4}$ En estos escritos son agrupados por la edición académica bajo el título de De summa rerum siguiendo una propuesta del propio Leibniz (véase A VI, 3, 472). Sobre el problema de una posible aproximación sistemática a estos escritos, véase Parkinson, G. H. R., "Leibniz's De Summa Rerum: a Sistematic Approach", en Studia Leibnitiana 18, 1986, pp. 132-151. 
opúsculos tienen un carácter anómalo y es un verdadero desafío hacerlos cuadrar en la evolución general de la filosofía leibniziana, puesto que en ellos Leibniz experimenta con distintas soluciones extremas a fin de dar respuesta a algunos interrogantes fundamentales.

La relación de Leibniz con el atomismo constituye un verdadero enigma para los intérpretes contemporáneos. Aun cuando a lo largo de toda su obra pueden encontrarse fuertes críticas a esta teoría, él mismo confiesa en sus escritos maduros haber abogado por ella en su juventud. ${ }^{5}$ En armonía con esas declaraciones, algunos exégetas han intentado rastrear posiciones cercanas al atomismo en los textos leibnizianos redactados entre 1666 y 1676. Al respecto pueden hallarse interpretaciones muy dispares; ellas van desde quienes, como Beeley, niegan cualquier tipo de compromiso real con los átomos y afirman que todas las referencias a ellos se reducen a meras especulaciones privadas que Leibniz nunca consideró seriamente, hasta quienes, como Robinet, parecieran considerar que la teoría leibniziana de los indivisibles de 1671 puede leerse en clave atomista. ${ }^{6}$ Todos concuerdan, no obstante, en que después de Paris Leibniz abandona cualquier inclinación hacia esta posición filosófica. Ahora bien, el compromiso más claro con el atomismo se encuentra en los textos pertenecientes a su último año en la capital francesa, tales como Notas sobre ciencia y metafísica (1676), Sobre el secreto de lo sublime o sobre lo más elevado de todas las cosas (1676) y Sobre la plenitud del mundo (1676), Sobre las verdades, la mente, Dios y el universo (1676) y Una cadena de maravillosas demostraciones acerca del universo (1676).

\footnotetext{
5 Véase GP IV, 478.

6 Véase Beeley, P., "Reponse to Arthur, Mercer, Smith and Wilson", en Leibniz Society Review, $\mathrm{n}^{\circ}$ 7, 1997, pp. 65-82; Robinet, A., Architectonique disjonctive, automates systémiques et idéalité transcendentale dans l' cuvre de G. W. Leibniz, Paris, Vrin, 1986, pp. 185-189. Para un resumen del problema del atomismo, así como de sus principales interpretaciones, véase Arthur, R., “The Enigma of Leibniz's Atomism”, Oxford Studies in Early Modern Philosophy, 1, 2004, pp. 183-228. Es conveniente indicar que Robinet sólo habla del "modelo atomista del punto indivisible de Cavalieri" y de la imposibilidad de afirmar átomos físicos una vez que se rechazan los indivisibles. Arthur presenta una crítica doble a esta posición: en primer lugar, los indivisibles no se identifican con los átomos físicos porque en la comprensión leibniziana de los mismos no son cuerpos, sino puntos inextensos y, en segundo lugar, el mayor compromiso de Leibniz con el atomismo es en 1676, año en el que ya no se aceptan los indivisibles, los cuales se abandonan en De minimo et maximo (1673).
} 
En estos ensayos Leibniz propone una variante particular de atomismo: no se trata ni del atomismo clásico, ni de la recuperación que hace Gassendi a comienzos de siglo XVII, ni menos aún del atomismo cartesiano defendido por Cordemoy, sino que estamos frente a una versión original y, al mismo tiempo, sumamente problemática. En líneas generales, Leibniz propone que es necesario que en el pleno de cuerpos fluidos haya átomos esféricos, cada uno de los cuales genere un vórtice al que le está asociada una mente determinada. Entre las diversas tesis sostenidas y abandonadas por Leibniz, ésta constituye sin duda la más curiosa, desconcertante y contraria tanto a su pensamiento anterior como posterior. A pesar de ello, el atomismo leibniziano de De summa rerum constituye un hito en su pensamiento que ayuda a comprender el desarrollo general de su metafísica. En el presente trabajo defendemos una doble hipótesis. Por una parte, sostenemos que el atomismo leibniziano recibe su inspiración fundamental de la cosmología cartesiana. Por otra parte, argumentamos que esta propuesta se realiza con el fin de dotar a los cuerpos de sustancialidad. ${ }^{7} \mathrm{~A}$ fin de lograr ambos cometidos dividiremos el trabajo en dos momentos. En primer lugar, estudiamos qué entiende Leibniz por átomo y cómo argumenta en su favor. En segundo lugar, evaluamos la deuda que esta propuesta tiene con el cartesianismo y analizamos su conexión con el problema general de la sustancia corpórea.

\section{2. Átomos, pleno físico y vacío metafísico}

El argumento a favor de los átomos toma como punto de partida un principio que, desde el período parisino en adelante, Leibniz comienza a utilizar con frecuencia en sus escritos de metafísica. En Sobre el secreto de lo sublime es presentado en los siguientes términos: "establezco por principio la armonía de las cosas, es decir, que cuanta más esencia pueda existir, existe” (A VI, 3, 472).

7 En nuestro trabajo discutimos fundamentalmente con la interpretación de Wilson, quien plantea una triple hipótesis respecto del atomismo leibniziano de 1676: primero, surge del encuentro entre el materialismo hobbesiano con el panteísmo spinozista; segundo, vuelve imposible la pluralidad de sustancias; tercero, constituye el primer gran antecedente del concepto de mónada. Véase Wilson, C., "Atoms, Minds and Vortices in De Summa Rerum: Leibniz vis-à-vis Hobbes and Spinoza", en S. Brown, The Young Leibniz and bis Philosopby (1646-1676), Dordrecht, Kluwer, 1999, pp. 223-243. 
Este principio, que podríamos llamar principio de armonía, es metafísico en la medida en que regula la existencia de las cosas, al igual que, por ejemplo, el principio de razón suficiente y el de identidad de los indiscernibles. En líneas generales, establece que existe la mayor cantidad de esencia posible. En un breve opúsculo del mismo año, Leibniz lo expresa en estos términos: "cualquier cosa que puede existir y es compatible con las restantes, existe" (A VI, 3, 582). ${ }^{8}$ Esta segunda formulación manifiesta una mayor claridad en la medida en que permite observar la doble utilidad del principio de armonía. Por una parte, su aporte negativo radica en restringir el ámbito de lo real: el paso de la posibilidad a la existencia está limitado por el hecho de que, tal como declara en Mi principio es que cualquier cosa que puede existir y es compatible con las restantes, existe (1676), "no todos los posibles pueden existir por sí junto con los restantes" (A VI, 3, 581). ${ }^{9}$ En este breve opúsculo Leibniz precisa cómo entender el principio de armonía en términos de composibilidad, reemplazando la noción de individuo posible por la de mundo posible, según el cual cada individuo se piensa intrínsecamente interconectado con la totalidad de los otros individuos en el mundo que configuran. Asimismo, Leibniz afirma que el principio de armonía es un criterio racional para determinar cuál de estas series de posibles pasará a la existencia, pues "la razón de existir de todos los posibles no debe ser limitada más que por lo que no es totalmente compatible; y así, no hay otra razón para determinar la existencia de los posibles a no ser que existan otros más perfectos, esto es, que envuelvan mayor realidad" (A VI, 3, 582). Por otra parte, el principio de armonía tiene un uso positivo que consiste en

8 Sobre este tema, véase Lloyd, G., "Leibniz on Possible Individuals and Possible Worlds", en Australasian Journal of Philosophy, vol. 56, n² 2, 1978, p. 127. Si bien Leibniz no desarrolla cómo opera la elección divina, en este breve ensayo expone el núcleo capital de su teodicea madura, a saber, que Dios sigue un principio independiente de Él en la determinación de lo posible como existente.

${ }^{9}$ Tal como plantea Lloyd, con el principio de armonía Leibniz combate las posturas de corte necesitaristas, puesto que el objetivo del mismo no es sino limitar el vínculo entre posibilidad y existencia. Lloyd afirma que el motivo fundamental de Leibniz para ello es objetar tesis como las spinozistas, en las cuales todo lo que está en el pensamiento de Dios ha de hacerse actual, desapareciendo así cualquier brecha entre lo posible y lo existente. Véase Ibid., p. 126. 
garantizar la mayor realidad posible para el mundo. ${ }^{10}$ En efecto, la consecuencia inicial que Leibniz extrae de este principio es la plenitud del mundo:

A partir de este principio se sigue que no hay ningún vacío en las formas y que no hay ningún vacío en el lugar y el tiempo, en la medida en que eso es posible. De ello se sigue que no hay ningún tiempo asignable en el que no haya algo, ni lugar alguno que no esté lleno en la medida en que es posible. Hay que estudiar, por consiguiente, qué se sigue a partir de la plenitud del mundo (A VI, 3, 472-473).

Una de las mayores curiosidades del atomismo leibniziano radica en que, como veremos a continuación, los átomos se derivan de la plenitud del mundo, esto es, se infieren de su teoría antitética por excelencia. La prueba de la existencia de los átomos guarda la siguiente estructura: [i] se muestra que el mundo tiene mayor realidad o esencia con átomos que sin ellos; [ii] se prueba que son posibles; [iii] se concluye que existen por el principio de armonía y, a partir de allí, se extraen algunas consecuencias.

Comencemos precisando qué entiende Leibniz por átomo. En los textos de 1676 los átomos son definidos en estos términos: "cuerpos sólidos" (A VI, 3, 473), "cuerpos inseparables" (A VI, 3, 524), "una porción de materia que es sólida e irrompible [...], un cuerpo singular inseparable" (A VI, 3, 393). El átomo no se concibe como algo sin partes, sino como una fracción de materia cuyas partes son inseparables o indivisibles. ${ }^{11}$ Por ellos, son cuerpos

10 "A fin de incrementar la multitud de cosas no es necesario una pluralidad de mundos, pues no hay ningún número de cosas que no estén en este único mundo y, ciertamente, en cualquiera de sus partes" (A VI, 2, 581).

${ }^{11}$ En este punto podría evidenciarse un problema en la propuesta leibniziana, en la medida en que los átomos son concebidos como cuerpos extensos indivisibles, pero desde 1672 en adelante el propio Leibniz rechaza la existencia de indivisibles en general. Sobre este punto, véase Robinet, Architectonique disjonctive, automates systémiques et idéalité transcendentale dans l' cuvre de G. W. Leibniz, pp. 186-189. Creemos que el concepto de indivisible que Leibniz vincula con los átomos no es el mismo que defendiera en 1671. En ambos casos se caracterizan por tener partes inseparables o indivisibles. Sin embargo, el indivisible de 1671 se caracteriza por ser algo de una cantidad inasignable, más pequeña que cualquier cantidad dada (de allí que los piense como inextensos), mientras que el átomo, como veremos más adelante en trabajo, se define por tener una 
infinitamente rígidos, que se diferencian del resto de los cuerpos porque, aun cuando tienen una extensión determinada, no pueden descomponerse en cuerpos más pequeños. De hecho, la única diferencia entre un átomo y otro parece reducirse a su cantidad, pues no hay variaciones de formas para Leibniz y es cuanto menos problemático afirmar diferencias cualitativas entre ellos. En principio, el concepto de algo extenso indivisible es contrario a la concepción leibniziana de la extensión que mantiene tanto en su juventud como en su madurez. Leibniz es consciente de eso también en 1676 y, como veremos más adelante, el hiato entre ambos conceptos es una de las razones que esgrime para introducir una mente asociada con cada átomo, las cuales serán las responsables de darle indivisibilidad a las porciones de extensión y así constituir los átomos.

Dejando por el momento la dificultad recién mencionada, es preciso comprender por qué Leibniz afirma que un mundo con átomos sería más perfecto que uno sin ellos. En Sobre el secreto de lo sublime sostiene que:

En primer lugar, probaremos que es necesario que además de los cuerpos fluidos también existan los cuerpos sólidos. Ellos son, en efecto, más perfectos que los fluidos, porque contienen más esencia; sin embargo, no todas las cosas pueden ser sólidas, pues entonces se obstaculizarían mutuamente; por consiguiente, los sólidos están mezclados con los fluidos (A VI, 3, 473).

El punto de partida de Leibniz radica en que los átomos o cuerpos sólidos contienen más esencia que los cuerpos fluidos. Concedido el punto, las posibilidades que surgen son, entonces, que sólo haya cuerpos fluidos, que sólo existan los cuerpos sólidos o que existan tanto unos como otros. La primera opción se rechaza por el principio de armonía, pues tal mundo contendría menos esencia que uno que, además de cuerpos fluidos, también tuviese cuerpos sólidos, dado que éstos son, se supone, superiores en ese respecto a los fluidos. Pero, si se admitiera lo segundo, esto es, que entonces sólo hay sólidos, la imperfección que sobrevendría sería la de que todos ellos se estorbarían mutuamente. Por tanto, sólo cabe que existan ambos tipos. Ahora

cantidad asignable (no es algo más pequeño que cualquier cantidad dada). En este caso, por tanto, Leibniz parece pensar una suerte de inseparabilidad de facto de sus partes que se diferencia del concepto de indivisible tomado de Cavalieri en su juventud. 
bien, cabe señalar que esta tesis es problemática, pues el supuesto inicial, según el cual un cuerpo sólido, esto es, de partes inseparables, tiene mayor realidad o esencia que uno fluido, es decir, de partes separables, lejos está de ser algo evidente incluso en el marco del planteo leibniziano. De hecho, si nos atenemos a un criterio puramente cuantitativo de la perfección o esencia, no parecería agregar nada el hecho de que las partes de una porción de materia sean inseparables. No es manifiesto que un cuerpo sólido contenga más esencia que uno fluido. Tomemos un cuerpo sólido y uno fluido que ocupen un espacio similar, ¿por qué el primero tiene más esencia? ¿Quiere decir con ello que contiene más materia un átomo que un cuerpo fluido? Esto, empero, no sería así según la propia concepción leibniziana de los cuerpos en estos años, ya que la separabilidad o no de las partes no suma nada a la esencia del cuerpo ni hace que haya más materia en uno que en otro. ${ }^{12} \mathrm{~A}$ fin de entender la tesis leibniziana, podría pensarse que el criterio de perfección abarca una dimensión cualitativa, según la cual la realidad del mundo aumenta con la variedad de sus componentes. En tal caso podría aceptarse que es más perfecto un mundo que tenga cuerpos fluidos y también sólidos antes que uno con sólo cuerpos del primer tipo. No obstante, esta lectura no parece ser la idea que tiene Leibniz en estos escritos.

Como segundo paso de la prueba, Leibniz tiene que mostrar que los átomos son posibles. Éste es, sin duda, el punto más áspero de la argumentación. En Sobre el secreto de lo sublime presenta la cuestión a partir del siguiente interrogante: "¿es acaso congruente con la razón que existan átomos?" (A VI, 3, 473). El desafío que debe afrontar Leibniz en este caso es demostrar que la teoría atómica es compatible con la tesis plenista. En principio, reconoce que no es posible que haya únicamente pleno y átomos, pues en tal caso no podría explicarse el movimiento. Es necesario introducir algún elemento adicional. Con tal fin, Leibniz introduce la distinción entre pleno físico y vacio metafísico, el cual es equiparado al que en la tradición atomista se

${ }^{12}$ Dado que Leibniz carece de conceptos como el de densidad, se sigue que, si dos esferas, una sólida y otra fluida, ocupan un mismo espacio, ellas tendrían la misma cantidad. La explicación leibniziana requiere de alguna precisión extra que, sin embargo, el filósofo no ofrece en estos escritos. 
denominara vacio intersticial ${ }^{13}$. En Sobre la plenitud del mundo, presenta un esquema gráfico que ilustra esta distinción y, al mismo tiempo, permite entender por qué considera que los átomos han de ser esféricos:

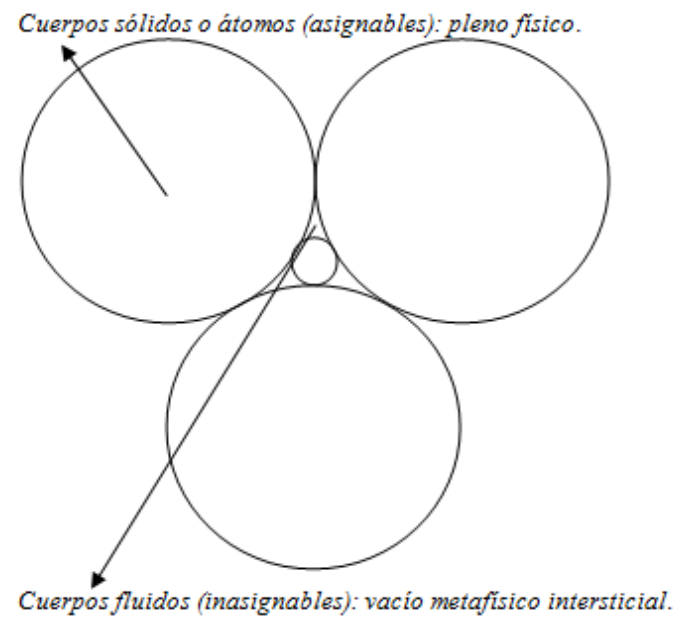

La solución leibniziana consiste en plantear que hay tantos átomos o cuerpos sólidos como puntos asignables en el espacio puedan pensarse y que los cuerpos fluidos son, en verdad, menores que cualquier cuerpo asignable. De esta forma, habrá tantos átomos como se quiera, pues en cada punto del espacio es asignable uno nuevo, y, sin embargo, con cada esfera introducida se genera un intersticio entre ellas que no se puede eliminar por más que agregue

${ }^{13}$ La fuente del filósofo alemán de este concepto es Gassendi, quien, siguiendo a los clásicos, distingue entre el vacio extramundano (externo al mundo, como el existente entre los planetas), el vacio intersticial (entre las partes de la materia del mundo) y el vacio coacervado o acumulado (creado por medios mecánicos). 
otras esferas. ${ }^{14}$ En Sobre el secreto de lo sublime afirma un vínculo entre este vacío metafísico y los cuerpos fluidos en la medida en que:

parece seguirse de la presencia de lo sólido en el fluido que la materia perfectamente fluida no es otra cosa más que la multitud de puntos infinitos, es decir, de cuerpos menores que lo que puede ser asignado, o sea, que se da necesariamente un vacío metafísico intersticial, lo que no está en contradicción con el pleno físico" (A VI, 3, 473, sub. prop.).

El carácter esférico de los átomos que conforman el pleno es presupuesto por Leibniz a fin de poder introducir este tipo vacío. Si fuesen, por ejemplo, poliedros, podrían tocarse sin dejar espacio libre entre ellos, lo que dificultaría la explicación del movimiento. En suma, Leibniz quiere marcar que en el ámbito físico todo es pleno, porque no hay punto asignable donde no haya cuerpos sólidos, pero, como no se puede eliminar el intersticio entre los átomos sin importar su número, hay asimismo un vacio metafísico (inasignable). Es por ello que juzga que los átomos no son contrarios a la tesis plenista y, por lo tanto, que son posibles. ${ }^{15}$

${ }^{14}$ En Sobre la plenitud del mundo afirma: "cada vez estoy más y más convencido de que los cuerpos inseparables, en tanto no surgen del movimiento, deben ser simples y, por ello, esféricos, pues todas las otras formas están sujetas a variación. Por lo tanto, parece indubitable que existen infinitos átomos esféricos. Si no hubiera átomos, entonces, dado el pleno, todas las cosas se disolverían. Un maravilloso pleno de este tipo que expongo es racional, incluso cuando no consiste en nada más que en esferas. Pues no hay lugar más pequeño que no pueda asumirse que existe en él una esfera más pequeña. Aceptando que las cosas son así, no habría ningún lugar asignable vacío" (A VI, 3, 524525).

${ }^{15}$ En Sobre el secreto de lo sublime resume su tesis del siguiente modo: "El pleno físico puede mantenerse con un vacío metafísico no asignable. Quizás de aquí se sigue que la materia está dividida en puntos perfectos, es decir, en todas las partes en las que puede ser dividida [...]. Por consiguiente, la materia es un ente discreto, no continuo, sólo contiguo, y está unido por el movimiento o por cierta mente" (A VI, 3, 473-474). "Es verdad que cualquier parte de materia, por pequeña que sea, contiene una infinidad de creaturas, es decir, es un mundo, se sigue también que la materia está actualmente dividida en una infinidad de puntos. Y esto es verdadero, porque es posible" (A VI, 3, 474). 
Una vez demostrado que los átomos introducen una mayor perfección en el mundo y, además, que son posibles, como tercer y último paso Leibniz concluye, en virtud del principio de armonía, que los átomos existen. No obstante, su defensa del atomismo no culmina en este punto, pues estas entidades le brindan la ocasión para argumentar, al igual que hiciera en su juventud, en favor de la existencia de otros elementos, a saber, mentes o principios incorpóreos, entidades que le permitirán ensayar una nueva propuesta respecto de la sustancialidad de los cuerpos. A ello nos dedicaremos en la segunda sección del trabajo.

\section{Vórtices, mentes y sustancia corpórea}

La primera consecuencia que Leibniz extrae de su propuesta es que los átomos reclaman un principio incorpóreo o mente particular que le esté asociado a cada uno. En sus escritos presenta dos razones para establecer tal conclusión. En primer lugar, juzga que la unidad de los átomos, esto es, su inseparabilidad o solidez, no es algo que tenga la materia misma o que pueda explicarse por el solo movimiento. Sin embargo, como estos cuerpos extensos indivisibles existen (hecho que juzga haber demostrado a partir del argumento antes visto), es necesario algún principio distinto del cuerpo que le otorgue su solidez:

He establecido sobre otras bases que hay alguna porción de materia que es sólida e irrompible $[. .$.$] y, como, además, la conexión no puede ser explicada$ en términos de solo materia y movimiento, como creo que se muestra en otro lugar satisfactoriamente, se sigue que el pensamiento ingresa en la formación de esa porción [de materia] y que, cualquiera sea su cantidad, deviene un cuerpo que es singular e inseparable, esto es, un átomo, siempre que tenga una mente singular (A VI, 3, 393).

La mayor dificultad de esta tesis radica en que Leibniz no desarrolla explícitamente cómo se produce tal unión ni tampoco cómo el principio incorpóreo le brinda solidez al cuerpo. Sin embargo, si atendemos al tipo particular de acción que la mente viene a introducir en los átomos, creemos que su propuesta puede esclarecerse, al menos en parte. 
En efecto, Leibniz asocia las mentes con un movimiento particular que se genera en el pleno: los vórtices. La razón de esta conexión no es explicitada por el filósofo alemán, pero puede reconstruirse una explicación si se toman algunos elementos básicos de la teoría cosmológica defendida por Descartes, que parece servir como fuente de inspiración para el modelo atomista pergeñado por Leibniz. En Sobre el secreto de lo sublime, el filósofo alemán plantea que "si algún cuerpo grande que se resiste a su disolución se moviese en el fluido, inmediatamente formaría una especie de pequeña esfera y un vórtice" (A VI, 3, 473).

En sintonía con el planteo cartesiano, Leibniz sostiene que la existencia de cuerpos sólidos en un medio fluido genera un movimiento de vórtice alrededor suyo, lo cual, en conjunción con el principio de inercia, da por resultado un movimiento circular. ${ }^{16}$ Este tipo de movimiento tiene primacía en las teorías plenistas en la medida en que es el único movimiento inicial posible. Ahora bien, la principal dificultad que tiene introducir cuerpos en rotación para un moderno radica en el componente antinatural que supone. En particular, el movimiento rotacional que da origen a un vórtice a su alrededor no es algo que se siga ni de la esencia de los cuerpos (de la cual sólo se sigue para el joven Leibniz la movilidad, esto es, la capacidad de moverse, pero no el movimiento efectivo) ni tampoco de los cuerpos ya puestos en movimiento (de lo cual sólo se sigue el movimiento rectilíneo). De allí que Leibniz, al igual que hiciera Descartes, asocie el movimiento de vórtices con un principio distinto de los cuerpos: en el caso del pensador francés tal principio es Dios, que pone en

16 "Cada mente tiene una duración ilimitada. Cada mente también está indisolublemente implantada en alguna materia, la cual tiene una cantidad definida. Cada mente tiene un vórtice alrededor suyo" (A VI, 3, 476-477). En textos como Sobre el origen de las formas (1676), Leibniz afirma que no sólo el cuerpo necesita a la mente, sino también las mentes a los cuerpos, pues "de hecho, la mente es agregada a una materia y sin la materia no percibiría como lo hace" (A VI, 3, 519). En efecto, Leibniz caracteriza ya en estos años a las mentes por su percepción (esto es, por llevar a la unidad una multiplicidad), para lo cual necesita de una constitución determinada en su cuerpo. Sin explicitar por qué, Leibniz juzga en 1676 que los vórtices podrían servir para ello. Sobre el vínculo entre el vórtice y la percepción de las mentes, véase Wilson, "Atoms, Minds and Vortices in De Summa Rerum: Leibniz vis-à-vis Hobbes and Spinoza”, pp. 238-240. 
rotación algunos cuerpos celestes para dar inicio a la maquinaria del mundo, pero en el caso del pensador alemán se adopta una versión más radical, donde hay tantos vórtices como átomos y, además, tantas mentes particulares como vórtices. Leibniz no pareciera sino aplicar - de un modo bastante precipitadoel esquema astronómico de Descartes a cada uno de los átomos del universo, ${ }^{17}$ lo que resulta en una tesis cuanto menos problemática, pues no es evidente que tal infinitud de vórtices sea posible.

Nuestro objetivo no es analizar la validez de esta propuesta, que no es más que un bosquejo de teoría que es abandonada en poco tiempo, sino evaluarla en función del desarrollo de la metafísica leibniziana de los cuerpos. En particular, creemos que con este atomismo Leibniz busca dar respuesta a un interrogante que recorre toda su metafísica: el de la sustancialidad del cuerpo. Si bien en sus escritos juveniles Leibniz creyó haber dado con una solución apropiada, hacia finales del período parisino es consciente de que los principales pilares sobre los que se sostenían ya han caído y, por ello, se ve obligado a ofrecer una vía alternativa. Creemos que el atomismo de 1676 responde a este interrogante.

En sus años de juventud (1663-1672) Leibniz desarrolla una primera teoría del cuerpo, la sustancia y la sustancia corpórea que puede esquematizarse del siguiente modo. En primer lugar, el cuerpo es caracterizado como materia que existe en el espacio y, en virtud de ello, cuenta con dos notas características: la extensión y la impenetrabilidad. ${ }^{18}$ En segundo lugar, la sustancia en general se

17 "Parece haber una especie de centro de todo el universo y un vórtice infinito general y también cierta clase de mente perfectísima o Dios. Esta mente existe cono un alma total en la totalidad del cuerpo del mundo" (A VI, 3, 474). "Además, es necesario que haya tantos vórtices como cuerpos sólidos en la naturaleza, únicamente por su movimiento. $\mathrm{Y}$ hay tantas mentes o pequeños mundos o percepciones como hay vórtices en el mundo" (A VI, 3, 393).

18 Respecto de la definición general de cuerpo, hasta 1672 Leibniz defiende que "el cuerpo es eso que está en el espacio, esto es, la cosa coextensa al espacio" (A VI, 2, 167) y que "la materia es el ente que existe en el espacio o el ente coextenso al espacio" (A II, 1, 34). Por una parte, el espacio se define por su extensión: "el ente primero extenso o cuerpo matemático, que evidentemente no contiene más que tres dimensiones y es el lugar universal de todas las cosas" (A II, 1, 34). Por otra parte, la materia se caracteriza por su impenetrabilidad: "La materia es el ser extenso en segundo 
define como aquello que tiene en sí el principio de su acción. ${ }^{19}$ En tercer lugar, dado que la única acción que el joven Leibniz reconoce para los cuerpos es cambiar su lugar en el espacio, esto es, moverse, la sustancia corpórea se define como aquello que tiene en sí el principio del cambio locativo. ${ }^{20}$ En cuarto lugar, y mostrando la filiación con el mecanicismo moderno, el cuerpo se concibe como algo que por sí mismo es indiferente al movimiento: en la materia no hay ni tendencia al movimiento ni al reposo, sino que ello reclama siempre una

grado o ese que además de extensión o cuerpo matemático posee también un cuerpo físico, es decir, resistencia, antitipia, espesor, la propiedad de llenar un espacio, la impenetrabilidad, que consiste en eso por lo cual, aunque llegue otro ser parecido, es necesario que uno u otro ente ceda el lugar" (A II, 1,34). De allí que el cuerpo sea pensado con esas dos notas primitivas. En el marco de nuestra investigación es interesante señalar la gran cercanía que la concepción del cuerpo del joven Leibniz guarda con la propia de Gassendi, quien constituye una de las mayores influencias en sus años de juventud. Véase Lolordo, A., Pierre Gassendi and the Birth of Early Modern Philosophy, Cambridge, Cambridge University Press, 2006, pp. 100-129; Moll, K., Der junge Leibniz. II: Der Übergang vom Atomismus zu einem mechanistischen Aristotelismus. Der revidierte Anschluss an Pierre Gassendi, Stuttgart, Bad Cannstatt, 1982, pp. 128-129.

${ }^{19}$ En Sobre la transubstanciación (1668), Leibniz defiende, en sintonía con la tradición escolástica, que "(1) Sustancia es el ente subsistente por sí; (2) ente subsistente por si es lo que tiene el principio de acción en sí. En efecto, el ente subsistente por sí o esta o aquella sustancia asumida en el individuo es el sustrato (los escolásticos, en efecto, tenían la costumbre de definir el sustrato como lo que tiene el principio de acción en sí o actúa). Por lo tanto, el ente por sí subsistente tiene el principio de acción en sí. Q. E. D" (A VI, 1, 508)

${ }^{20}$ En su juventud Leibniz acepta que el único cambio del cuerpo es el movimiento y que a partir de él hay que explicar cualquier otra de sus modificaciones: "Toda acción y pasión del cuerpo es movimiento (...). Toda acción y pasión es ejercicio de potencia. El ejercicio de una potencia es tránsito de la potencia al acto o de la posibilidad no existente a la existente. La potencia del cuerpo está contenida en la esencia del cuerpo. La esencia del cuerpo es existencia en el espacio. La potencia (o posibilidad carente de existencia) que está contenida por la existencia en el espacio es la potencia de existir en otro espacio (igual y similar a aquel en el que ahora existe). La potencia de existir en otro espacio es mutabilidad de espacio. La mutabilidad de espacio es movilidad. El ejercicio de la movilidad es movimiento. Por lo tanto, toda acción del cuerpo es movimiento" (del tercer borrador a la Teoría del movimiento abstracto: A VI, 2, 168). 
causa externa. ${ }^{21}$ A partir de estos elementos se evidencia cuál es el problema de la sustancia corpórea en la filosofía del joven Leibniz: dado que el cuerpo no es capaz de dar cuenta de su acción, esto es, de su movimiento, por sí solo no es sustancia. Con vistas a dotar a los cuerpos de sustancialidad, Leibniz propone entre 1668 y 1672 una teoría hilemórfica de la sustancia corpórea según la cual con cada cuerpo concurre una mente particular que oficia como principio de su movimiento. ${ }^{22}$

El hilemorfismo que Leibniz defiende hasta 1672 contiene, además de las ideas básicas antes enumeradas, una serie de tesis subyacentes que son requisitos indispensables para su propuesta: la noción de movimiento y espacio absoluto, la concepción de los indivisibles como cantidades cuyas partes son indistantes y constituyen los límites de las cantidades continuas (tales como la extensión), la caracterización del conato como el indivisible del movimiento y, por último, la comprensión de la mente como un punto de concurrencia de conatos contrarios. Ahora bien, ninguna de estas tesis particulares sobrevive a los cambios de Paris. Y es por ello que la particular solución juvenil al problema de la sustancialidad de los cuerpos no se encuentra entre ninguna de las muchas teorías defendidas en De summa rerum. Sin embargo, este contexto de crisis permite dar sentido a la teoría atomista que Leibniz ensaya en 1676. En efecto, el esquema de átomos, vórtices y mentes asociadas, a pesar de su rareza, ofrece una respuesta alternativa frente a la pregunta sobre la sustancialidad de los cuerpos sin apelar a ninguna de las tesis juveniles abandonadas. Asimismo, esta propuesta guarda una semejanza estructural con la propuesta juvenil que ha de destacarse: se trata nuevamente de una teoría hilemórfica en la que los cuerpos

${ }^{21}$ En la Confesión de la naturaleza contra los ateístas (1668) argumenta que "La movilidad se sigue de la naturaleza del cuerpo, pero no el movimiento mismo" (A VI, 1, 490). La misma idea es reiterada en la carta a Thomasius de abril de 1669, donde afirma nuevamente que de la extensión e impenetrabilidad se sigue la movilidad, pero no el movimiento (véase A II, 1, 36). La escisión entre las nociones de cuerpo y movimiento constituye una de las notas distintivas de la filosofía moderna y es una tesis que Leibniz mantiene a lo largo de toda su vida: "es necesario que la causa del movimiento sea externa al cuerpo" (A II, 1, 19).

${ }^{22}$ En este sentido declara en Sobre la transubstanciación que "ningún cuerpo, separado de la mente concurrente, tiene el principio de movimiento en sí. (...). Por lo tanto, ningún cuerpo, separado de la mente concurrente, es sustancia. (A VI, 1, 508-509) 
(extensos e impenetrables al igual que en su juventud, pero ahora además inseparables) reclaman un principio incorpóreo (inextenso) para garantizar su unidad y movimiento. Por ello, creemos que el atomismo leibniziano de 1676 ha de leerse como un intento por dotar a los cuerpos nuevamente de sustancialidad, siguiendo para ello un esquema similar al propuesto en 1672: no es sino el último intento de recuperar el esquema general de metafísica juvenil, el cual será abandonado definitivamente en los años posteriores a Paris. ${ }^{23}$

Recibido: 04/2017; aceptado: 07/2017

${ }^{23}$ Sobre este tema, véase Fazio, R., "Leibniz y la reforma de la metafísica: un análisis de la definición de sustancia como fuerza primitiva activa", en Revista Latinoamericana de Filosofía, Vol. XLII, Nº 2, 2016, pp. 149-170. 